\title{
ROUTE OPTIMIZATION IN MANETS WITH ACO AND GA
}

\author{
Pankaj Vidhate ${ }^{1}$, Yogita Wankhade ${ }^{2}$ \\ ${ }^{1}$ Lecturer, IT dept., S.G.R.E.F's C.O.E.M, Ahmdnagar, Maharashtra, India, pankajvidhate@gmail.com \\ ${ }^{2}$ Lecturer, IT dept., S.G.R.E.F's C.O.E.M, Ahmednagar, Maharashtra, India, yogita16.wankhade@gmail.com
}

\begin{abstract}
A mobile ad-hoc network consists of a collection of mobile nodes which can communicate with each other with the help of wireless links without the help of any pre-existing communication infrastructure. Due to the lack of infrastructure in these type of networks, nodes itself can act as a routers and relay the packets from source to destination. There are so many routing protocols used in MANET which also maintains connectivity from source to destination when links on these paths are break due to some causes like node movements, radio propagation, drainage of battery, and wireless interference. Routing is one of the important issues which are having a significant impact on network performance. Different measures which are concerned with the Quality of Service are like end to end delay, packet delivery ratio, control overhead, pause time, routing overhead, and distance in between source and destination pair. Different optimization techniques can be used to find out an available optimal path from source to destination. In this paper, we are using Ant Colony Optimization for finding out best possible paths, along with Genetic Algorithm which helps in giving the globally optimal solution from all the best possible paths which were produced by Ant colony optimization. This proposed algorithm called as GA-API overcomes the delay in packet delivery by producing the shortest path and also overcomes the problem of communication interruption due to node or link failure by finding multiple paths between pair of source and destination nodes.
\end{abstract}

Index Terms: MANET, Genetic Algorithm, Ant colony Optimization, API.

\section{INTRODUCTION}

A mobile ad-hoc network consists of a collection of mobile nodes which can communicate with each other with the help of wireless links without the help of any pre-existing communication infrastructure. Nodes within each other's radio range communicate directly via wireless links, while those that are far apart rely on intermediate nodes to forward their messages. Mobile ad hoc networks are the selforganizing and self-configuring wireless networks consisting of multiple numbers of hops. MANET is made up of a set of mobile hosts which can freely movable and helps in delivering or relaying packets on behalf of one another. Due to the lack of infrastructure in these type of networks, nodes itself can act as a routers and relay the packets from source to destination. For deploying MANETs, there is no need of infrastructure resulting into the use of MANETs in the scenarios like crowd control, search and disaster rescue operations and battlefields.

Routing is one of the important issues which are having a significant impact on network performance. These routing protocols are playing a vital role in deciding Quality of Service. Different measures which are concerned with the Quality of Service are like end to end delay, packet delivery ratio, control overhead, pause time, routing overhead, and distance in between source and destination pair. One can improve the Quality of Service by reserving the resources which would be used in the path in between source and destination pair for packets delivery, by minimizing delay required by a packet to reach to its destination, by reducing the probability of link failures or providing an alternate arrangement of path in case of link or node failure, also by providing security for the nodes which are being used in the Mobile ad hoc network formation as well as for the nodes which newly wants to join to the already existed Mobile ad hoc network. Quality of Service for the routing protocols also deals with an optimization of the routing paths.

Different optimization techniques can be used to find out an available optimal path from source to destination. It is not necessary that the optimal path should be always the shortest one, but it should the feasible path which gives the best possible path from source to destination. In this paper, we are using Ant Colony Optimization for finding out best possible paths, along with Genetic Algorithm which helps in giving the globally optimal solution from all the best possible paths which were produced by Ant colony optimization. It means ACO provides best possible solution and GA prevents the algorithm from going into the local optima.

\section{RELATED WORK}

\subsection{Introduction}

The highly dynamic nature of a mobile ad hoc network results in frequent and unpredictable changes of network topology, adding difficulty and complexity to routing among the mobile nodes. The challenges and complexities, coupled with the critical importance of routing protocol in establishing communications among mobile nodes, make the routing area the most active research area with the MANET domain. 
Numerous routing protocols and algorithms have been proposed. The performance of these protocols has been studied and compared under different types of networks or network environments and also under different traffic conditions. Number of different survey papers and comparative analyses of different mobile ad hoc routing protocols have been published.

Routing of the packets in the MANET has several issues to be cope up with, like host mobility, rate of frequent link failures, rate of link repairs. The rate of link failures and repairs may be high when nodes move fast [1].

Busola S. Olagbegi and Natarajan Meghanathan presented a review on energy efficient and secure multicast routing protocol for Mobile ad hoc network. A multicast routing protocol is used to support the transmission of data from a sender to all the receivers of a multicast group by using the available bandwidth more efficiently, while topology is frequently changing. Multicasting is improving the efficiency of the wireless link when it is sending multiple copies of messages. Secure multicast routing is also playing an important role in Mobile ad hoc networks. They have reviewed different energy efficient routing protocols like Energy efficient Location Aided Routing (EELAR), Power Aware Localized Routing (PLR), Minimum Energy Routing Protocol (MER), etc [2].

Ashwani Kush and Sunil Taneja take an effort to provide Secured Routing over MANET with Power Management. In this paper hashing and power estimation is done for route request, reply and not in route error and route erasure phases. So that less routing overhead occurs [3].

\subsection{Ant Colony Optimization}

The Quality of Service of routing protocols can also be enhanced by minimizing the routing overhead. Different optimization techniques can be used for that, Ant Colony Optimization is one of them.

The ACO is based on the behavior of foraging of different ants. When ants are searching for their food, these ants wander randomly in any direction and after finding food or resource they get returned to their nest position or colony. While wandering and coming back to their colony ants are laying some chemical substance along the path or route which is called as pheromone. Many ants can take different routes for reaching to the same food source. The ants, which take or use the shortest path, reinforce that path with more quantity of pheromone which helps other ants to follow that shortest path. In the mean time, more number of other ants are getting attracted because of this pheromone trail, which makes the path even stronger. This nature of ants is helpful in quickly identifying the shortest path. Ants are nothing but autonomous agents which are interacting through an indirect communication which is also known as stigmergy, where stigmergy is one of the indirect forms of communication in which these individual ant agents leave signals in the environment. These signals are then being sensed by some other agents to decide their own way of behaving.

S. Prasad, Y.P. Singh, and C.S. Rai has proposed Probabilistic Ant Routing algorithm which is based on Ant Colony Optimization. The Probabilistic Ant Routing algorithm is a proactive routing protocol and it uses both pheromone and heuristic value [4].

Gianni Di Caro, Frederick Ducatelle and Luca Maria Gambardella have worked on AntHocNet. AntHocNet is a hybrid multipath algorithm, designed by considering the principles of Ant Colony Optimization routing. AntHocNet consists of both proactive and reactive components. It does not maintain routes to all possible destinations at all times like the original ACO algorithms for wired networks, but only sets up paths from source to destination when they are needed while starting of a data session [5].

\subsection{Genetic Algorithm}

Genetic Algorithms is branch of different computational models which gets its inspiration from the human evolution. The solution for a specific problem is being encoded by these genetic algorithms on a chromosome like data structure. Then after recombination operators are being applied to these structures. In this way genetic algorithms preserve critical information. Genetic algorithms can be used as a function optimizer, although the ranges of these problems are very broad to which these algorithms have been applied. With a random population of different chromosomes an implementation of genetic algorithm is begun. After this evaluation of these structures and the allocated reproductive opportunities has been allocated to different chromosomes which are representing good solution for the defined problem are given more chances for reproducing themselves as compared to the chromosomes which are producing poorer solutions. The goodness of a solution is decided with the help of current available population.

C. Safak Sahin, Ibrahim Hokelek, and Giorgio Bertoli have presented a force based genetic algorithm [6]. This algorithm is for self-spreading mobile nodes uniformly over a geographical area. In this algorithm, each mobile node exchanges its genetic information of direction and speed encoded in its chromosomes with its neighboring nodes.

D. Suresh Kumar, K. Manikandan and M.A. Saleem Durai have worked on secure and adaptive backup routing protocol for mobile ad hoc networks [7]. In the cases of failure of links these discovered paths are used. To improve quality of service of routing, size of the buffer, end to end required delay and shortest possible paths are used as parameters for GA in route discovery.

Damla Turgut, Sajal K. Das, Ramez Elmasri and Begumhan Turgut have worked on a paper representing the usefulness 
of the genetic algorithms for increasing the performance of the clustering algorithms in ad hoc networks [8].

\section{PROPOSED SCHEME}

In Mobile ad hoc networks due to its dynamic topology changes, there is always a requirement of route discovery between the pair of nodes. Due to the mobile nature of the nodes and the different constraints like node failure, link failure, energy issues of the nodes plays a vital role in the quality of service of routing algorithm. Because of these factors it is always necessity to find out the possibilities of the further routes present in between the pair of nodes. Ant colony optimization will be very helpful to optimize the possible available route in between the pair of source and destination. The optimization in the route discovery and route selection is required all the time in mobile ad hoc networks.

This paper introduces a new algorithm which does the hybridization in between API algorithm (one of the type of ant colony optimization) and a real coded genetic algorithm (RCGA). If API is compared to its ACO surrogates, who were mainly applied to discrete optimization problems, API is designed for the continuous optimization problems. The proposed ant-base algorithm is different from the basic ant colony optimization in terms of search strategy. In this proposed ant-base algorithm, the ants have to travel with the help of their memory which stores visual landmarks which were encountered along with the familiar routes, rather than using pheromones as used in ACO algorithms. Mainly, API focuses at maximizing the prey rather than minimizing the path. The drawback of API is that it may be ending up quickly in a solution which is a local minimum. The reason behind ending up quickly is due to a regular movement of the nest which is only in the best position found by the ants. API is used to find out the solution for the continuous optimization problems although it could stop at local minima, it is useful to find out the solutions which are close to the globally optimal.

In mobile ad hoc networks due to its dynamic topology changes, there is always a requirement of route discovery between the pair of nodes. Different factors like link failure, node failure, node battery backup leads to the continuous changes in the mobile ad hoc network causing to find out all the possible routes available in between source and destination pair so that if one route gets failed there would be the another possible route available. In case of the higher dimension mobile ad hoc networks, there is a large number of mobile nodes are available. So number of routes can be available, out of these available routes, the route which is optimal one should be get selected for the packet delivery. So the Genetic algorithm creates a population of all these routes in between the pair of nodes. From this population of routes the optimal route is getting selected with the help of Ant colony optimization.

Thus, ant colony optimization algorithms' percentage of best solutions (say K \%) are added to the Genetic Algorithm population pool after which the breeding process is followed proportional to their fitness. Then after in this ACO search process another percentage is added which is nothing but a percentage of the best individuals in the Genetic Algorithm (say L \% of the GA population) which gives "fitnessproportional pheromone". As like the percentage of best individuals, another percentage of worst fitted individuals of Genetic algorithm (say $\mathrm{M} \%$ ) are used to "evaporate a constant amount of pheromones" in the Ant Colony Optimization search. The migration would not be taken place when either algorithms find an improvement or both algorithms do no improve after iteration.

This paper proposes a hybrid algorithm which stochastic in nature, intending to solve global unconstrained continuous optimization problems. These problems can be of medium and large dimensions. With the help of a hybridization of the two classes of evolutionary optimization algorithms an algorithm is proposed in this paper. The two classes of evolutionary optimization algorithms are as follows: a special class of Ant Colony Optimization algorithm for continuous domain and a Real-Coded Genetic Algorithm.

The proposed algorithm is entitled as GA-API, which keeps the downhill search ability of API. Meanwhile the local minimum trapping in the API algorithm is avoided by using diversity in the solution which is given by Real-Coded Genetic Algorithm. Due to the use of proposed optimization tool the computational time is get reduced also increases the robustness and consistency of high quality approximate solutions.

\section{STUDY AND ANALYSIS OF INPUT AND OUTPUT TO THE PROJECT}

\section{Input to API:}

API is one of the surrogates of ant colony optimization algorithm. The mobile ad hoc network information is given to this API algorithm. This API algorithm uses this information of all nodes and the links present in between these nodes and traces out the feasible paths in between the source and destination pairs. As the nodes in MANETs are mobile in nature, they are continuously changing their positions causing the link breakage and new link formation all the time. This behavior of mobility changes the information provided to the API algorithm, and API has to trace out all the available routing paths for this changing behavior.

\section{Input to GA:}

Genetic algorithm basically works with the large quantity of input. With this input genetic algorithm forms its own population which is nothing but the solution space for the given problem. In this project, the real coded genetic algorithm is used. The output from the API is provided to this RCGA, which further creates new routing paths from these routes. These routing paths will leads to the formation 
of population. From this population, by using different genetic operators like crossover, mutation etc, next offspring are created which are again included in the population. Out of these solutions present in the solution then the good ones are forwarded to the API algorithm again, so that API algorithm will not get stuck to the local optima because of its local solution space.

Input to GA-API:

GA-API algorithm is a combination of Genetic algorithm and API algorithm. The output from the API algorithm is provided to the GA-API algorithm for further improvement. The solutions are track out by GA-API which was provided by API algorithm. From these solutions the solution which one is globally optimal is been selected as the optimized solution or the optimized path from source to destination.

Output Analysis:

The output given by the GA-API algorithm is an optimized path for routing in between the pairs of source and destination. The output is analyzed with the help of different parameters. The parameters used for analysis are such as end to end delay required for packet delivery, packet delivery ratio in between the given source and destination pair, bandwidth consumed while transferring packets, the amount of battery gets consumed during the communication. By using all these measures it get decided that whether the route selected by GA-API algorithm is the optimum one or not.

\section{CONCLUSIONS}

In this paper, a new algorithm, called as GA-API is proposed which is a combination of Genetic Algorithm along with API algorithm which is nothing but one of the surrogate of Ant Colony Optimization algorithm. This algorithm is proposed to solve global unconstrained continuous optimization problems. By combining some unique characteristics of two robust meta-heuristic algorithms such as RCGA and API, the hybrid metaheuristic algorithm is proposed in this paper. GA-API provides satisfactory or optimum solutions, with much less computational effort. This algorithm works well for large, complex problems with greater dimensionality. GA-API gave near global solutions. But GA-API failed to find the global optimum solution in some cases like for the optimization problems which are generally of small dimensions.

\section{ACKNOWLEDGEMENT}

I would like to acknowledge all the great people who have been assisted me right the way through my research work. I would like to thank my parents and my friends for encouragement and suggestions at every time for this research. The acknowledgement would be unfinished without talk about of the lucky thing of the almighty.

\section{REFERENCES}

[1]. Josh Broch, David Maltz, David Johnson, Yih-Chun Hu, and Jorjeta Jetcheva, "A performance comparison of multihop wireless ad hoc network routing protocols" in MobiCom'98, ACM, 1998.

[2]. Busola Olagbegi and Natarajan Meghanathan, "A Review of the Energy Efficient \& Secure Multicast Routing Protocols For MANETs" in International Journal on applications of graph theory in Wireless ad hoc networks, vol. 2, No. 2, June 2010.

[3]. Ashwani Kush and Sunil Taneja, "Secured Routing over MANET with Power Management" in ACM, 2011.

[4]. S.Prasad, Y.P. Singh, and C.S. Rai, "Swarm based intelligent routing for MANETs" in International Journal on Recent Trends in Engg., Vol. 1, No. 1, May 2009.

[5]. Gianni Di Caro, Frederick Ducatelle and Luca Maria Gambardella, "AntHocNet: An Adaptive Nature-Inspired Algorithm for Routing in Mobile Ad Hoc Networks" in IDSIA, 2004.

[6]. C. Safak Sahin, Ibrahim Hokelek, and Giorgio Bertoli, "Genetic Algorithms for Self-Spreading Nodes in MANETs" in GECCO'08 ACM 2008.

[7] D. Suresh Kumar, K. Manikandan and M.A. Saleem Durai, "Secure On-Demand Routing Protocol for MANET using Genetic Algorithm" in International Journal of Computer Applications Vol. 19, No. 8, April 2011.

[8]. Damla Turgut, Sajal K. Das, Ramez Elmasri and Begumhan Turgut, "Optimizing Clustering Algorithm in Mobile Ad hoc Networks Using Genetic Algorithmic Approach",2000.

\section{BIOGRAPHIES}

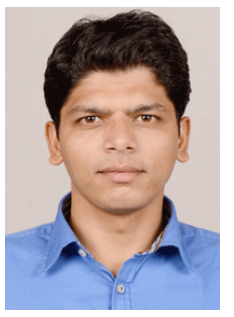

Pankaj Vidhate received the Bachelor's degree in Information Technology engineering from University of Pune, India in 2009 and currently working for M.E. degree in Computer Networks Engineering from University of Pune, India.

$\mathrm{He}$ is currently lecturer with the SGREF COE Ahemednagar, University of Pune. His research interests include optimization problems in mobile ad hoc networks

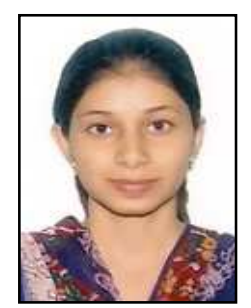

Yogita Wankhade received the Bachelor's degree in Information Technology engineering from University of India in 2009 and currently working for M.E. degree in Computer Networks Engineering from University of Pune, India 